\title{
Epidemiologia das fraturas diafisárias de tíbia em um hospital municipal de referência em traumatologia
}

\section{Epidemiology of tibial diaphyseal fractures in a reference municipal hospital in traumatology}

Sidnei Torres Vieira Júnior ${ }^{1}$. Antônio Pierre Aguiar Júnior² ${ }^{2}$ Luis Pimentel Sombra ${ }^{3}$. Juvêncio Oliveira Araújo de Castro ${ }^{4}$. Francisco Robson de Vasconcelos Alves ${ }^{4}$.

1 Médico Residente de Ortopedia e Traumatologia do Hospital Infantil Albert Sabin, Fortaleza, Ceará, Brasil. 2 Médico Residente de Ortopedia e Traumatologia do Hospital Geral de Fortaleza, Ceará, Brasil. 3 Médico Residente de Ortopedia e Traumatologia do Hospital Universitário Walter Cantídio (HUWC), Fortaleza, Ceará, Brasil. 4 Médico ortopedista do Instituto Doutor José Frota, Fortaleza, Ceará, Brasil.

\section{RESUMO}

Objetivos: descrever o perfil epidemiológico de pacientes com fratura diafisária de tíbia tratados em um serviço de trauma em um hospital municipal terciário e comparar com a literatura nacional. Métodos: todos os pacientes admitidos neste hospital com fratura de tíbia no período de janeiro a março de 2015. Dados clínicos e sociodemográficos foram colhidos de um sistema eletrônico de registros da própria instituição. Foi realizada análise descritiva padrão, com cálculo de média e desvio padrão para variáveis numéricas e distribuição de frequência para variáveis categóricas. A análise bivariada foi realizada através do teste qui-quadrado para variáveis categóricas e Mann Whitney e Kruskal-Wallis para variáveis numéricas. Foram considerados estatisticamente significantes, valores de p menores ou iguais a 0,05 . Resultados: dos 123 pacientes avaliados, 85,2\% eram do sexo masculino e $14,8 \%$, do sexo feminino. A idade média foi de 32,4 anos. No período, 122 fraturas expostas foram diagnosticadas. O tipo de fratura 42-A foi o mais prevalente (41,3\%) seguido pelo tipo 42-B (34,8\%) e 42-C (23,9\%). As fraturas associadas mais comuns foram as do fêmur proximal $(11,1 \%)$ e do platô tibial (11,1\%). A estabilização temporária com fixador externo foi o tratamento adotado em todos os pacientes no departamento de emergência (100\%). Para o tratamento definitivo, a osteossíntese com placa e parafusos foi o mais utilizado (37,5\%). A média de internamento hospitalar foi de 24,6 dias, que é estatisticamente relacionada à quantidade de fraturas associadas. Conclusão: os pacientes tratados nessa instituição apresentam o perfil epidemiológico semelhante àquele encontrado na literatura nacional.

Palavras-chave: Centros de traumatologia. Fraturas ósseas. Epidemiologia.

\section{ABSTRACT}

Purpose: to develop an epidemiological study of diaphyseal tibial fractures in patients treated in a trauma service in a local municipal tertiary hospital. Methods: all patients admitted in the hospital with tibial fracture from January to March of 2015. Sociodemographic and clinical data were collected from electronic system registration of patients of the institution. Standard descriptive analysis was performed, with mean and standard deviation calculation for numerical variables and frequency distribution for categorical variables. The bivariate analysis was performed using the chi-square test for categorical variables and Mann Whitney and Kruskal-Wallis for numerical variables. p values less than or equal to 0.05 were considered statistically significant. Results: of the 123 patients who were evaluated, $85.2 \%$ were male and $14.8 \%$ were female. The mean age was 32.4 years. During this period, 122 open fractures were diagnosed. The 42-A type fractures were the most frequent (41.3\%) followed by the type $42-\mathrm{B}(34.8 \%)$ and $42-\mathrm{C}(23.9 \%)$. The most common associated fractures were the fractures of the proximal femur and tibial plateau (11.1\%). The temporary stabilization with external fixator was the adopted treatment in all patients at the emergency department (100\%). As a definitive treatment the osteosynthesis with plate with screws was the most used (37.5\%). The mean hospital stay was 24.6 days, which is statistically related to the amount of associated fractures. Conclusion: patients treated at this institution have an epidemiological profile similar to those found in a national literature.

Keywords: Trauma centers. Fractures, bone. Epidemiology.

Autor correspondente: Sidnei Torres Vieira Júnior, Avenida Beira Mar, 1407, apartamento 2120, Meireles, Fortaleza, Ceará. CEP: 60165-121. Telefone: +55 85 3263-2322. E-mail: sidnei66@hotmail.com

Conflito de interesses: Não há qualquer conflito de interesses por parte de qualquer um dos autores.

Recebido em: 25 Jul 2016; Revisado em: 12 Dez 2016; Aceito em: 08 Fev 2017. 


\section{INTRODUÇÃO}

O trauma constitui, atualmente, um grave problema de saúde pública, uma vez que é responsável por importante parcela dos atendimentos hospitalares em todo o mundo. Dos pacientes, vítimas de trauma fechado, $85 \%$ sofrem lesão do sistema músculo esquelético. ${ }^{1}$ Por muitos anos, a tíbia foi o osso longo mais fraturado, ${ }^{2}$ sendo as fraturas do terço médio as mais frequentes. ${ }^{3}$ As fraturas de tíbia apresentam o maior número de opções de tratamento ${ }^{4,5}$ e a classificação mais utilizada tem sido a da Orthopaedic Trauma Association/Arbeitsgemeinschaft für Osteosynthesefragen (OTA/AO), que leva em consideração a região do osso acometido, a energia e o mecanismo de trauma e classifica as fraturas simples em A, as fragmentadas com cunha em B e as multifragmentadas complexas em C. ${ }^{6}$ Essa classificação permite uma boa diferenciação e entendimento do padrão da fratura, com boa relação com o prognóstico e resultado clínico. ${ }^{7}$ Também permite algum entendimento da lesão associada das partes moles, mas como isso não faz parte da classificação, permite que, se o cirurgião não estiver atento, faça somente a classificação da fratura, sem a devida correlação com a lesão de partes moles. ${ }^{8}$

Devido ao desenvolvimento das técnicas de osteossíntese, facilitando o ato cirúrgico e diminuindo os seus riscos, a abordagem cruenta no tratamento dessas lesões tem se destacado na preferência dos ortopedistas..$^{9,10} \mathrm{O}$ uso de fixadores externos, placas e parafusos, e haste intramedular bloqueada para a estabilização das fraturas da tíbia são descritas na literatura. ${ }^{11-15}$

As fraturas de tíbia podem ser fechadas ou expostas. As fraturas expostas são particularmente difíceis de tratar devido à pobre cobertura de partes moles e reduzido suprimento sanguíneo em algumas regiões. Esse diagnóstico indica um tratamento cirúrgico de urgência, com desbridamento, antibioticoterapia e estabilização óssea. ${ }^{16}$

Estudos epidemiológicos são fundamentais para desenvolver um melhor entendimento a respeito das moléstias ao sistema músculoesquelético, auxiliando nas medidas preventivas e terapêuticas.

O objetivo deste estudo é avaliar o perfil epidemiológico e sociodemográfico dos pacientes internados no serviço de traumatologia de um hospital terciário municipal do Sistema Único de Saúde (SUS) com fratura diafisária de tíbia.

\section{CASUÍSTICA E MÉTODOS}

Trata-se de um estudo retrospectivo, descritivo e analítico, realizado no serviço de traumatologia de um hospital terciário municipal do SUS da cidade de Fortaleza. Os critérios de inclusão utilizados foram todos os pacientes admitidos no serviço no período de janeiro a março de 2015, que foram submetidos a tratamento cirúrgico ou conservador das fraturas de diáfise tibial e que constavam no sistema de cadastro eletrônico de pacientes desse serviço. Os critérios de exclusão utilizados foram os pacientes que não possuíam dados e/ou exames registrados no sistema de cadastro eletrônico. Um formulário foi elaborado e preenchido pelos autores, contendo informações sociodemográficas e clínicas, como: idade, sexo, procedência, tempo de internação hospitalar, lado da fratura, fraturas associadas, classificação das fraturas e tipo de tratamento realizado.

O banco de dados dessa pesquisa foi construído com o auxílio do Microsoft Excel. Posteriormente, os dados foram levados para o Statistical Package for the Social Science (SPSS) para análise estatística. Foi realizada análise descritiva padrão, com cálculo de média e desvio padrão para variáveis numéricas e distribuição de frequência para variáveis categóricas. A análise bivariada foi realizada através do teste qui-quadrado para variáveis categóricas e Mann Whitney e Kruskal-Wallis para variáveis numéricas. Foram considerados estatisticamente significantes valores de $\mathrm{p}$ menores ou iguais a 0,05 .

O estudo foi previamente aprovado pelo Comitê de Ética em Pesquisa da Instituição.

\section{RESULTADOS}

Os dados relacionados às características sociodemográficas encontram-se na Tabela 1. O estudo envolveu a participação de 123 pacientes: $85,2 \%$ eram do sexo masculino e $14,8 \%$ do sexo feminino. A idade dos pacientes variou de 7 a 82 anos, com média de 32,4 anos. Em relação à procedência, 52,6\% eram da capital e $47,4 \%$ eram de cidades do interior ou da região metropolitana.

As características clínicas também encontram-se expostas na Tabela 1. Do total de 123 fraturas avaliadas, 122 (99,2\%) foram expostas, sendo $7(5,7 \%)$ destas por arma de fogo. Apenas uma fratura fechada foi avaliada. Fraturas associadas ocorreram em 34 pacientes $(27,6 \%)$, sendo que $11,1 \%$ eram fraturas do fêmur proximal, 11,1\% eram fraturas do platô tibial, seguidas em ordem decrescente por: fraturas diafisárias do rádio ou da ulna, fraturas distais do rádio ou da ulna, fraturas diafisárias do fêmur e fratura do acetábulo ou do anel pélvico (Tabela 1).

Tabela 1. Características sociodemográficas e clínicas de pacientes com fratura diafisária de tíbia. Intituto Dr José Frota, Fortaleza-CE-janeiro a março 2015.

\begin{tabular}{lll}
\hline & $\mathrm{N}$ & $\%$ \\
\hline Procedência & & \\
$\quad$ Capital & 61 & 52,6 \\
$\quad$ Interior/região metropolitana & 55 & 47,4 \\
Sexo & & \\
$\quad$ Masculino & 98 & 85,2 \\
Feminino & 17 & 14,8 \\
Tipo de fratura & & \\
Exposta & 122 & 99,2 \\
Fechada & 1 & 0,8 \\
\hline
\end{tabular}

Continua 
Conclusão

Tabela 1. Características sociodemográficas e clínicas de pacientes com fratura diafisária de tíbia. Intituto Dr José Frota, Fortaleza-CEjaneiro a março 2015.

\begin{tabular}{|c|c|c|}
\hline & $\mathrm{N}$ & $\%$ \\
\hline Presença de lesões associadas & 34 & 27,6 \\
\hline \multicolumn{3}{|l|}{ Local das lesões associadas } \\
\hline Ulna/radio disfisário & 4 & 8,9 \\
\hline Ulna/radio distal & 4 & 8,9 \\
\hline Ossos do carpo & 1 & 2,2 \\
\hline Quirodáctilos & 3 & 6,7 \\
\hline Acetábulo/anel pélvico & 4 & 8,9 \\
\hline Fêmur proximal & 5 & 11,1 \\
\hline Fêmur disfisário & 4 & 8,9 \\
\hline Fêmur distal & 1 & 2,2 \\
\hline Platô tibial & 5 & 11,1 \\
\hline \multicolumn{3}{|l|}{ Classificação AO } \\
\hline $42-\mathrm{A} 1$ & 10 & 10,9 \\
\hline $42-\mathrm{A} 2$ & 16 & 17,4 \\
\hline $42-\mathrm{A} 3$ & 12 & 13,0 \\
\hline $42-B 1$ & 8 & 8,7 \\
\hline $42-B 2$ & 18 & 19,6 \\
\hline $42-\mathrm{B} 3$ & 6 & 6,5 \\
\hline $42-\mathrm{C} 1$ & 2 & 2,2 \\
\hline $42-\mathrm{C} 2$ & 7 & 7,6 \\
\hline $42-\mathrm{C} 3$ & 13 & 14,1 \\
\hline \multicolumn{3}{|l|}{ Tratamento de emergência } \\
\hline Fixador externo linear & 90 & 100,0 \\
\hline \multicolumn{3}{|l|}{ Tratamento definitivo } \\
\hline Fixador externo linear & 10 & 15,6 \\
\hline Ilizarov & 04 & 6,3 \\
\hline Placa DCP & 24 & 37,5 \\
\hline Placa em ponte & 13 & 20,3 \\
\hline Haste intramedular & 13 & 20,3 \\
\hline
\end{tabular}

Quanto à classificação $\mathrm{AO}$, os tipos de fraturas mais encontradas foram as fraturas de traço simples (42-A), correspondendo a $41,3 \%$ dos casos. As fraturas fragmentadas com cunha (42-B) corresponderam a $34,8 \%$ dos casos e $23,9 \%$ a fraturas multifragmentadas complexas (42-C).

Pacientes com maior idade apresentaram fraturas causadas por trauma de menor energia, de acordo com a classificação AO (Tabela 1).

A estabilização da fratura com uso de fixador externo linear foi o tratamento adotado na emergência em todos os casos, seja provisório ou definitivo.

Quanto ao tratamento definitivo, 37,5\% dos pacientes foram tratados com método de compressão interfragmentar, com uso de placa de compressão dinâmica (DCP) e parafusos. O tratamento com o uso de placa em ponte e haste intramedular foi adotado em 20,3\% dos pacientes, cada um. A osteossíntese com fixador externo linear foi adotada como tratamento definitivo em $15,6 \%$ dos pacientes. Dos pacientes do nosso estudo, $6,3 \%$ foram submetidos a tratamento definitivo com fixador externo circular tipo Ilizarov.

Como mostrado na Tabela 2, a média de dias de internação foi de 24,6 dias, sendo o mínimo de três e o máximo de 156 .

Tabela 2. Dias de internação em relação às variáveis sociodemográficas e clínicas de pacientes com fratura diafisária de tíbia. Intituto Dr José Frota, Fortaleza-CE-janeiro a março 2015.

\begin{tabular}{|c|c|c|c|c|c|}
\hline & Mínimo & Máximo & Média & $\begin{array}{l}\text { Desvio } \\
\text { padrão }\end{array}$ & $\mathrm{P}$ \\
\hline \multicolumn{6}{|l|}{ Procedência } \\
\hline Capital & 3 & 122 & 28,61 & 28,452 & 0,198 \\
\hline $\begin{array}{l}\text { Interior/ } \\
\text { região } \\
\text { metropolitana }\end{array}$ & 4 & 156 & 22,89 & 31,311 & \\
\hline \multicolumn{6}{|l|}{ Sexo } \\
\hline Masculino & 3 & 122 & 23,77 & 24,670 & 0,785 \\
\hline Feminino & 3 & 156 & 39,50 & 52,968 & \\
\hline \multicolumn{6}{|l|}{ Lado da fratura } \\
\hline Direito & 3 & 156 & 27,36 & 35,400 & 0,232 \\
\hline Esquerdo & 3 & 117 & 22,46 & 22,846 & \\
\hline Bilateral & 122 & 122 & 122,00 & - & \\
\hline \multicolumn{6}{|l|}{ PAF } \\
\hline Sim & 19 & 66 & 37,20 & 20,969 & 0,024 \\
\hline Não & 3 & 156 & 23,88 & 29,394 & \\
\hline \multicolumn{6}{|l|}{$\begin{array}{l}\text { Presença } \\
\text { de lesões } \\
\text { associadas }\end{array}$} \\
\hline Sim & 3 & 122 & 45,57 & 34,855 & $<0,001^{*}$ \\
\hline Não & 3 & 156 & 16,68 & 21,988 & \\
\hline \multicolumn{6}{|l|}{$\begin{array}{l}\text { Classificação } \\
\text { AO }\end{array}$} \\
\hline $42-\mathrm{A} 1$ & 6 & 67 & 39,29 & 24,831 & 0,053 \\
\hline $42-\mathrm{A} 2$ & 3 & 117 & 22,21 & 29,717 & \\
\hline $42-\mathrm{A} 3$ & 4 & 36 & 15,00 & 12,530 & \\
\hline 42-B1 & 7 & 43 & 17,71 & 11,658 & \\
\hline $42-B 2$ & 3 & 55 & 20,33 & 17,546 & \\
\hline $42-\mathrm{B} 3$ & 5 & 20 & 12,50 & 10,607 & \\
\hline $42-\mathrm{C} 1$ & 42 & 43 & 42,50 &, 707 & \\
\hline $42-\mathrm{C} 2$ & 7 & 156 & 75,29 & 58,531 & \\
\hline $42-\mathrm{C} 3$ & 8 & 53 & 27,00 & 14,852 & \\
\hline
\end{tabular}

Tratamento

definitivo

\begin{tabular}{llccll}
$\begin{array}{l}\text { Fixador } \\
\text { externo linear }\end{array}$ & 8 & 8 & 8,00 & - & 0,113 \\
Ilizarov & 25 & 156 & 101,00 & 67,978 & \\
\hline
\end{tabular}

Continua 
Conclusão

Tabela 2. Dias de internação em relação às variáveis sociodemográficas e clínicas de pacientes com fratura diafisária de tíbia. Intituto Dr José Frota, Fortaleza-CE-janeiro a março 2015.

\begin{tabular}{lllll}
\hline & Mínimo & Máximo & Média & $\begin{array}{l}\text { Desvio } \\
\text { padrão }\end{array}$ \\
\hline $\begin{array}{l}\text { Tratamento } \\
\text { definitivo }\end{array}$ & & & & \\
$\quad$ Placa DCP & 4 & 67 & 23,82 & 20,084 \\
$\quad \begin{array}{l}\text { Placa em } \\
\text { ponte }\end{array}$ & 16 & 66 & 30,56 & 18,501 \\
$\begin{array}{l}\text { Haste } \\
\text { intramedular }\end{array}$ & 3 & 118 & 41,50 & 47,936 \\
\hline
\end{tabular}

* p estatisticamente significante.

A relação entre a existência de lesões associadas e o tempo de internação foi estatisticamente significativo, com o tempo máximo de internação de 122 dias e média de 45,57 dias em pacientes com alguma fratura associada (Tabela 2).

Os pacientes com fratura por Projétil de Arma de Fogo (PAF) tiveram em média 37,2 dias de internação, enquanto que os pacientes sem fratura por PAF tiveram em média 23,8 dias de internação, porém sem significância estatística (Tabela 2).

Em relação à classificação $\mathrm{AO}$, os pacientes com fraturas do tipo A apresentavam média de 25,5 dias de internação hospitalar, as do tipo B 16,8 e as do tipo C 48,3 dias, porém, não houve significância estatística (Tabela 2).

Os pacientes submetidos a tratamento definitivo com fixador externo linear tiveram o menor tempo de internação hospitalar com média de 8 dias. Os pacientes tratados com placa DCP e parafusos tiveram média de 23,8 dias. Os tratados com placa em ponte e parafusos permaneceram em média 30,6 dias internados e os tratados com haste intramedular tiveram alta com média de 41,5 dias. Devido a fatores intrínsecos ao hospital estudado, os pacientes que necessitaram de fixador externo circular (Ilizarov) permaneceram internados em média 101 dias (Tabela 2).

Foi evidenciado, de acordo com a Tabela 3, que, dos pacientes tratados pelo método da compressão interfragmentar com placa DCP e parafusos, $26 \%$ eram classificados como tipo $42-\mathrm{A} 1(\mathrm{p}<0,001)$. Apenas fraturas do tipo 42-C2 ou 42-C3 foram tratadas por fixador externo circular (Ilizarov). O tratamento por haste intramedular não foi utilizado nos tipos de fratura em espiral (42- A1, B1 e C1).

Tabela 3. Classificação da fratura em relação ao tratamento definitivo de pacientes com fratura diafisária de tíbia. Intituto Dr José Frota, Fortaleza-CE-janeiro a março 2015.

\begin{tabular}{|c|c|c|c|c|c|c|c|}
\hline \multirow{2}{*}{$\begin{array}{l}\text { Classificação } \\
\text { AO }\end{array}$} & \multicolumn{5}{|c|}{ Tratamento Definitivo - N (\%) } & \multirow[t]{2}{*}{ Total } & \multirow[t]{2}{*}{$\mathrm{p}$} \\
\hline & $\begin{array}{l}\text { Fixador externo } \\
\text { linear }\end{array}$ & Ilizarov & Placa DCP & Placa em ponte & $\begin{array}{l}\text { Haste } \\
\text { intramedular }\end{array}$ & & \\
\hline 42-A1 & - & - & $4(26,7)$ & $1(7,7)$ & 0 & $5(9,4)$ & $<0,001$ \\
\hline $42-\mathrm{A} 2$ & $1(11,1)$ & - & $5(33,3)$ & - & $2(16,7)$ & $8(15,1)$ & \\
\hline $42-\mathrm{A} 3$ & $2(22,2)$ & - & $3(20,0)$ & - & $4(33,3)$ & $9(17,0)$ & \\
\hline $42-\mathrm{B} 1$ & $1(11,1)$ & - & - & $2(15,4)$ & - & $3(5,7)$ & \\
\hline 42-B2 & $2(22,2)$ & - & $2(13,3)$ & - & $2(16,7)$ & $6(11,3)$ & \\
\hline $42-B 3$ & $2(22,2)$ & - & $1(6,7)$ & - & $1(8,3)$ & $4(7,5)$ & \\
\hline $42-\mathrm{C} 1$ & - & - & - & $1(7,7)$ & - & $1(1,9)$ & \\
\hline $42-\mathrm{C} 2$ & - & $3(75,0)$ & - & - & $2(16,7)$ & $5(9,4)$ & \\
\hline $42-\mathrm{C} 3$ & $1(11,1)$ & $1(25,0)$ & - & $9(69,2)$ & $1(8,3)$ & $12(22,6)$ & \\
\hline Total & $9(17,0)$ & $4(7,5)$ & $15(28,3)$ & $13(24,5)$ & $12(22,6)$ & $53(100,0)$ & \\
\hline
\end{tabular}

* p estatisticamente significante.

\section{DISCUSSÃO}

Neste trabalho, inúmeros aspectos foram analisados com a finalidade de caracterizarmos as fraturas diafisárias da tíbia em nosso serviço. Essas informações são úteis para identificarmos algumas falhas que, porventura, possam estar acontecendo.

O perfil epidemiológico dos pacientes foi semelhante ao encontrado nos trabalhos nacionais e internacionais. ${ }^{17,18}$
A predominância de homens sobre mulheres e a idade dos pacientes na nossa amostra foram semelhantes aos evidenciados por Grecco et al. ${ }^{19}$ que evidenciaram uma proporção de homens sobre mulheres de 3:1, com a faixa etária mais acometida entre 21 e 30 anos.

Como em outros estudos, ${ }^{19}$ as fraturas de traço simples (42-A) foram as mais encontradas, já as fraturas com cunha (42-B) e multifragmentadas complexas (42-C) evidenciadas não foram semelhantes, entretanto, sem significância estatística. 
A modernização, a industrialização e o aumento da taxa de violência na sociedade têm contribuído para o crescimento da incidência das lesões traumáticas severas dos membros inferiores. ${ }^{23}$ A maior incidência em homens, jovens e em idade produtiva, demonstrada no presente estudo, relaciona-se diretamente a esses fatores, principalmente no que diz respeito ao mecanismo de lesão.

Pacientes com maior idade apresentaram fraturas causadas por trauma de menor energia, de acordo com a classificação AO, possivelmente relacionada a uma menor densidade mineral óssea.

O uso do fixador externo linear como tratamento na emergência em todos os casos, está relacionado à nossa amostra de 99,2\% de fraturas expostas. Entretanto, nossos dados divergiram da literatura, ${ }^{19}$ na qual $31,67 \%$ das fraturas expostas foram tratadas apenas com imobilização gessada; 54,83\% utilizou tratamento com fixador externo associado a imobilização gessada; $10 \%$ foram tratados apenas com placa e parafuso e $2,5 \%$, tratados unicamente com fixador externo. Estes dados nos revelam a real necessidade de avaliarmos melhor cada caso de fratura exposta da tíbia em nosso serviço, a fim de instituir logo na emergência o tratamento definitivo.

De acordo com estudos prévios, ${ }^{20}$ existem vários métodos de tratamento e muitas são as discussões favorecendo um ou outro. A haste intramedular bloqueada é considerada por muitos autores como método ideal para o tratamento das fraturas instáveis fechadas ou expostas da diáfise da tíbia, porém, de acordo com Hungria e Mercadante, ${ }^{21}$ nas fraturas expostas da diáfise da tíbia, a indicação da melhor forma de osteossíntese vai depender da gravidade da lesão óssea, do estado das partes moles e da idade do paciente.

Devido à pouca cobertura muscular da face medial da tíbia, é frequente a associação com extensas lesões de partes moles, como ocorre nas fraturas expostas da tíbia. O método de osteossíntese mais rápido, simples e que pode evitar maior lesão de partes moles é o fixador externo linear, o qual foi o tratamento mais instituído na emergência, sendo este empregado em 90 pacientes avaliados, seja provisório ou definitivo.

\section{REFERÊNCIAS}

1.Tscherne H, Gotzen L. Fractures with Soft Tissue Injuries. Berlin: Springer-Verlag Berlin Heidelberg; 1984.

2. Heckman JD, Sarasohn-Kahn J. The economics of treating tibia fractures. The cost of delayed unions. Bull Hosp Jt Dis. 1997;56(1):63-72.

3. Muller ME. Klassifikation und Internationale AO - Dokumentation der Femurfracturem. Unfallheilkunde. 1980;83:251-60.

4. Borges JL, Silva VC, Saggin JI. Haste intramedular bloqueada da tíbia. Rev Bras Ortop. 1997;32(1):46-50.

5. Ferreira JC. Fraturas da diáfise dos ossos da perna. Rev Bras Ortop. 2000;35(10):375-83.
Por se tratar de um hospital de referência em traumas complexos, apresentando casos com perda óssea importante e cobertura de partes moles prejudicada, 6,3\% das fraturas necessitaram ser tratadas com fixadores externos circulares do tipo Ilizarov.

Estudos relatam que o tempo de hospitalização está diretamente relacionado ao número de procedimentos cirúrgicos a que o paciente será submetido, ${ }^{22}$ nosso estudo comprovou ser estatisticamente significativo a relação entre a existência de lesões associadas e essa variável.

Os pacientes tratados definitivamente com fixador externo apresentaram menor tempo de hospitalização, já os pacientes submetidos a tratamento definitivo com osteossíntese interna passaram por um maior período de internação, refletindo a espera por uma maior resolução da cobertura de partes moles para a instituição de um tratamento definitivo com osteossíntese interna. Quanto ao longo período de internação dos pacientes que necessitaram de fixador externo circular (média de 101 dias) está relacionado ao tempo de acompanhamento até se obter uma melhora da cobertura de partes moles e ao pequeno número de cirurgiões aptos e disponíveis para instalar o Ilizarov neste serviço.

Esse estudo foi realizado no maior serviço de traumatologia do Estado do Ceará e conseguiu abordar as mais diversas situações clínicas e perfis socioeconômicos, contudo, devido ao curto período de coleta de dados (3 meses) não pudemos avaliar os resultados a longo prazo, como tempo necessário para a consolidação óssea e as possíveis complicações relacionadas à fratura de tíbia.

\section{CONCLUSÃO}

De maneira semelhante ao restante do cenário nacional, as fraturas diafisárias de tíbia da instituição estudada foram mais comuns em jovens do sexo masculino. Prevaleceram fraturas expostas em $122(99,2 \%)$ casos. O método de tratamento mais empregado na emergência foi o cirúrgico com fixador externo linear para estabilizar a fratura provisoriamente e a placa DCP e parafusos para tratamento definitivo. O tempo de internação relacionou-se com a quantidade de fraturas associadas.

6. Orthopaedic Trauma Association Committee for Coding and Classification. Fracture and dislocation compendium. J Orthop Trauma. 1996;10(Supl 1):1-154.

7. Swiontkowski MF, Agel J, McAndrew MP, Burgess AR, MacKenzie EJ. Outcome validation of the AO/OTA fracture classification system. J Orthop Trauma. 2000;14(8):534-41.

8. Kojima KE, Ferreira RV. Fraturas da diáfise da tíbia. Rev Bras Ortop. 2011;46(2):130-5.

9. Malta MC, Barreto JM. Fratura dos ossos da perna no adulto. In: Hebert S, Xavier R, Pardini AG Jr, Barros TE Filho. Ortopedia e traumatologia: princípios e prática. 3. ed. Porto Alegre: Artmed; 2003. p. $1376-87$. 
10. Bone LB, Johnson KD. Treatment of tibial fractures by reaming and intramedullary nailing. J Bone Joint Surg Am. 1986;68(6):87787.

11. Golubović Z, Stojiljković P, Macukanović-Golubović L, Milić D, Milenković S, Kadija M, et al. External fixation in the treatment of open tibial shaft fractures. Vojnosanit Pregl. 2008;65(5):343-8.

12. Della Rocca GJ, Crist BD. External fixation versus conversion to intramedullary nailing for definitive management of closed fractures of the femoral and tibial shaft. J Am Acad Orthop Surg. 2006;14(10):S131-5.

13. Agarwal A, Gulati D. Letter to the Editor: Immediate interlocking nailing versus external fixation followed by delayed interlocking nailing for Gustilo type IIIB open tibial fractures. J Orthop Surg (Hong Kong). 2007;15(2):393-4.

14. Park HJ, Uchino M, Nakamura M, Ueno M, Kojima Y, Itoman $\mathrm{M}$, et al. Immediate interlocking nailing versus external fixation followed by delayed interlocking nailing for Gustilo type IIIB open tibial fractures. J Orthop Surg (Hong Kong). 2007;15(2):131-6.

15. Ma CH, Wu CH, Tu YK, Lin TS. Metaphyseal locking plate as a definitive external fixator for treating open tibial fractures--clinical outcome and a finite element study. Injury. 2013;44(8):1097-101.

16. Rodrigues FL, de Abreu LC, Valenti VE, Valente AL, da Costa Pereira Cestari R, Pohl PH, et al. Bone tissue repair in patients with open diaphyseal tibial fracture treated with biplanar external fixation or reamed locked intramedullary nailing. Injury. 2014;45(Supl 5):S32-5.

17. Jaña FC Neto, Canal MP, Alves BA, Ferreira PM, Ayres JC, Alves $\mathrm{R}$. Analysis of the characteristics of patients with open tibial fractures of Gustilo and Anderson type III. Rev Bras Ortop. 2016;51(2):143-9.

18. Ali AM, McMaster JM, Noyes D, Brent AJ, Cogswell LK. Experience of managing open fractures of the lower limb at a major trauma centre. Ann R Coll Surg Engl. 2015;97(4):287-90.

19. Grecco MA, Prado I Jr, Rocha MA, Barros JW. Estudo epidemiológico das fraturas diafisárias de tíbia. Acta Ortop Bras. 2002;10(4):10-7.

20. Labronici PJ, Reis FB, Fernandes HJ. Estudo prospectivo do uso da haste intramedular bloqueada não fresada em fraturas fechadas e expostas da diáfise da tíbia. Clin Orthop. 2006;41(9):373-83.

21. Hungria JO, Mercadante MT. Osteossíntese provisória das fraturas expostas da diáfise da tíbia com fixador externo não transfixante. Rev Bras Ortop. 2008;43(1-2):31-40.

22.Balbachevsky D, Belloti JC, Martins CV, Fernandes, HJ, Faloppa F, Reis FB. Como são tratadas as fraturas expostas da tíbia no Brasil? Estudo transversal. Acta Ortop Bras. 2005:13(5):229-32.

23. Fodor L, Sobec R, Sita-Alb L, Fodor M, Ciuce C. Mangled lower extremity: can we trust the amputation scores? Int J Burns Trauma. 2012;2(1):51-8.

\section{Como citar:}

Vieira ST Júnior, Aguiar AP Júnior, Sombra LP, Castro JO, Alves FR. Epidemiologia das fraturas diafisárias de tíbia em um hospital municipal de referência em traumatologia. Rev Med UFC. 2017 set-dez;57(3):12-17. 